\title{
PROLIFERATING CELLS IN THE ADULT PRIMATE CEREBELLAR CORTEX AFTER ISCHEMIA
}

\author{
Vesselina Mihaleva ${ }^{1}$, Stoyan Pavlov ${ }^{1}$, Meglena Angelova ${ }^{1}$, Dessislava Marinova ${ }^{1}$, \\ Tetsumori Yamashima ${ }^{2}$, Vanya Goranova ${ }^{1}$, Anton B. Tonchev ${ }^{1}$ \\ ${ }^{1}$ Department of Anatomy, Histology and Embryology, Faculty of Medicine, \\ Medical University of Varna \\ ${ }^{2}$ Department of Restorative Neurosurgery, Medical Faculty, \\ University of Kanazawa, Japan
}

\begin{abstract}
INTRODUCTION: Brain ischemia is a devastating neurological condition with a significant medical and social impact. Here we investigated the effect of experimental ischemia of different duration on the ability of the adult macaque cerebellum to produce new cells of specific phenotypes.

MATERIALS AND METHODS: We used a well-established model of global brain ischemia in young adult Japanese monkeys applying bromodeoxyuridine (BrdU) for 5 days $x 100 \mathrm{mg} / \mathrm{kg}$ daily. The animals were distributed into different experimental and control groups depending on the postischemic survival periods: 4, 9, 15, 23, 44 days (D) and BrdU starting day. Immunohistochemical detection of BrdU+ cells, Iba1+ microglia and GFAP+ astroglia was performed on cryosections. Statistical evaluation of newly generated cells with phenotyping for microglia and astrocytes in various cortical layers of the cerebellum was done.

RESULTS AND CONCLUSIONS: The number of BrdU+ cells in some ischemic groups was significantly higher compared to control animals. In the cerebrocerebellum, there was an increasing value in the D4 group compared to the control, then slightly reducing in the D9 and D15 groups and increasing again in the D23 and D44 groups. In the spinocerebellum, an increase was detected only in the D44 group. The newly generated cells were dispersed in all cerebellar cortical layers with the highest concentration in the Purkinje cell layer. Our data show that ischemia stimulates cellular proliferation in the cerebellum but this effect declines with the time after ischemia. We found evidence for a generation of new microglia but not for astroglia. Our data may contribute to a better understanding of regeneration in the cerebellum after brain ischemia.
\end{abstract}

Keywords: global ischemia, primate cerebellum, BrdU/Iba1/GFAP immunohistochemistry

Address for correspondence:

Anton B. Tonchev

Department of Anatomy, Histology and Embryology

Medical University of Varna

55 M. Drinov Str.

9002 Varna, Bulgaria

e-mail:anton.tonchev@mu-varna.bg

phone: +35952677082

Received: July 19, 2016

Accepted: August 23, 2016

\section{INTRODUCTION}

Restricted brain regions of adult mammals contain neural stem/progenitor cells able to generate neurons and glia under physiological and pathological conditions. Two regions of the mammalian forebrain are shown to produce neurons that are successfully integrated in the existing local neuronal circuits (4). In states of injury such as ischemia, trauma and neurodegeneration these cells may proliferate and participate in reparative processes. The embryon- 
ic and early postnatal development of the mammalian cerebellum is intensively studied and many aspects are already well-defined $(2,3,6,7,12,15)$. Crucial cellular and molecular mechanisms are established mainly by the mouse cerebellum $(11,13,14)$. Regional expression of transcription factors in the cerebellar rhombic lip and ventricular zone shapes the fate of the hindbrain neuroepithelial stem cells of the developing cerebellum $(5,9,10,17,19)$. Developmental defects of the cerebellum are considered in relation to selected human neurological disorders (1). Many factors controlling tissue regeneration in the cerebellum after injuries still remain unclear. It is known that the murine postnatal cerebellum contains multipotent neural stem cells (8) but they do not produce functional neurons and astrocytes in adulthood (16). There is little information about proliferative events in the adult primate cerebellum that is evolutionary closer to human cerebellum.

In the present study we investigated the effect of global brain ischemia on cell proliferation and specification in the cerebellum of young adult macaques using the thymidine analog 5-bromo-2-deoxyuridine (BrdU), an indicator for DNA synthesis and cell proliferation. It is widely applied in studying neurogenesis, including the cerebellum $(14,16,19)$. We examined the number, distribution and some phenotypes of newly generated cells in various cortical layers of the adult primate cerebellum after global brain ischemia with different duration. Our results may be useful for an additional insight of regenerative processes in the cerebellum following ischemia by humans.

\section{MATERIALS AND METHODS}

Tissues from monkey brains were acquired at the Department of Restorative Neurosurgery at the Medical Faculty of the Kanazawa University in Japan in accordance with the guidelines of the institutional Ethics Committee and the NIH Guide for the Care and Use of Laboratory Animals. We used a well-established model of global brain ischemia in young adult Japanese monkeys (Macaca fuscata) and applied BrdU for 5 consecutive days x $100 \mathrm{mg} / \mathrm{kg}$ daily. Animals were distributed into various experimental and control groups depending on the survival postischemic period: 4, 9, 15, 23 and 44 days (D) and BrdU starting day, 2 animals in each group. In control animals (sham operation without ischemia) and $\mathrm{D} 4$ ischemic groups BrdU application started on day 0 , in the D9, D23 and D44 groups - on day 5 and in the D15 group - on day 11. Under total anesthesia, the monkeys were intracardially perfused with 4\% paraformaldehyde in 0.1 M PBS, pH 7.4. Brains were removed, tissue blocks were cut and postfixed for 2-3 h. After cryoprotection in 30\% sucrose and freezing, $40-\mu \mathrm{m}$-thick coronal cryosections were sequentially cut and stored at $-20^{\circ} \mathrm{C}$. Surgical and tissue procedures are presented in more details in Tonchev et al. (18). Standard single or double immunohistochemical peroxidase/fluorescence detection of newly generated cells and phenotyping for microglia with ionized calcium-binding adapter molecule 1 (Ibal)+ cells and astrocytes with glial fibrillary acidic protein (GFAP)+ cells was performed. To reveal incorporated BrdU, DNA was denatured in 50\% formamide $/ 2 \times \mathrm{SSC}$ (standard sodium citrate) buffer at $65^{\circ} \mathrm{C}$ for $2 \mathrm{~h}$ and then in $2 \mathrm{~N} \mathrm{HCl}$ at $37^{\circ} \mathrm{C}$ for $30 \mathrm{~min}$. Subsequently, the sections were washed in Tris-buffered saline containing $0.1 \%$ Triton X-100 (TBS-T). Nonspecific binding was blocked with TBS$\mathrm{T} / 10 \%$ normal horse or goat sera (TBS-TB) for 30 min, followed by incubation with the primary antibody diluted in TBS-TB for 2 days at $4^{\circ} \mathrm{C}$. The following primary antibodies and dilutions were used: rat anti-BrdU (1:100, Harlan Sera-Lab, Loughborough, UK), mouse anti-BrdU (1:100, Becton-Dickinson, San Jose, CA), rabbit anti-Ibal (1:800, gift from Dr. Y. Imai, Tokyo, Japan), and rabbit anti-GFAP (1:200, Sigma Chemical, St. Louis, MO).

For peroxidase immunohistochemistry, respective secondary anti-rat/anti-mouse IgG conjugated with biotin for $2 \mathrm{~h}$ was applied. Vector $\mathrm{ABC}$ kit (Vector Laboratories, Burlingame, CA) followed by diaminobenzidine (DAB, Sigma) as a chromogen was used. For immunofluorescence labeling, respective secondary goat anti-rat IgG-TRITC (Jackson Immunoresearch, West Grove, PA, USA), anti-mouse IgG-Alexa Fluor 488 or 546 and anti-rabbit IgG-Alexa Fluor 488 or 633 (Molecular Probes, Eugene, OR, USA), dilution 1:200, were applied for $2 \mathrm{~h}$ at room temperature.

Morphometric analysis was performed on digital images acquired with Axio Imager.72 Zeiss using AxioCam MRc and AxioVisioner software (Carl Zeiss Microscopy). Statistical quantification of 
Vesselina Mihaleva, Stoyan Pavlov, Meglena Angelova et al.

BrdU+ cells or evaluation of colocalization of BrdU/ Ibal and BrdU/GFAP markers was done in different cerebellar layers/zones: molecular layer, Purkinje cell layer, granular cell layer, and underlying white matter zone. For cell counting of BrdU+ cells, a digital frame $200 \times 200 \mu \mathrm{m}^{2}$ was applied to 10 adjacent fields in 5 different regions for each layer in each animal.
The mean numerical densities \pm standard deviation per group were determined and comparisons between groups were made. We applied descriptive statistics for creating diagrams and t-Test (Two-Sample Assuming Equal Variances) for comparison of values between groups. All countings were performed in a blinded fashion.
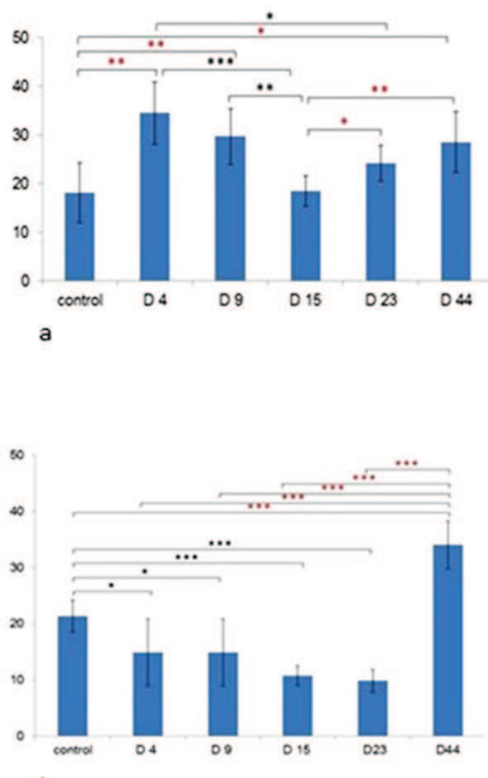

d

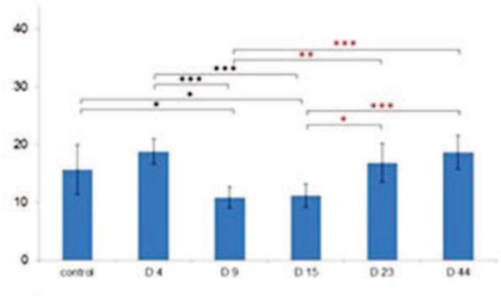

b

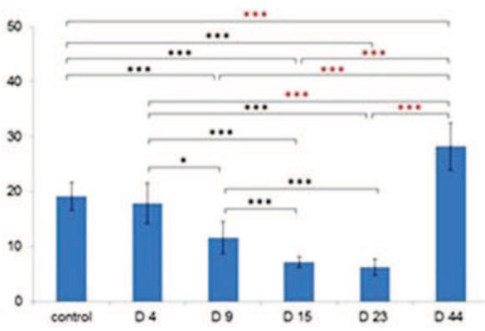

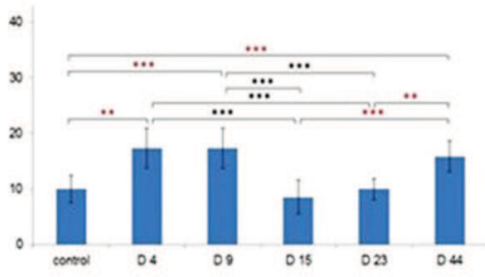

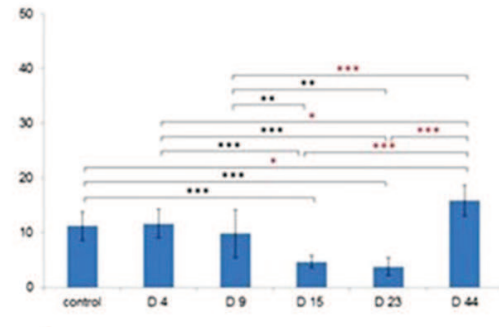

Fig. 1. Quantification of BrdU+cells in different layers/zones of the primate cerebrocerebellum $(a, b, c)$ and spinocerebellum $(d, e, f)$ : molecular layer $(a, d)$, granular layer $(b, e)$ and white matter zone $(c, f)$. Columns show mean numerical densities \pm standard deviation of cell counts in each group (control, D4, D9, D15, D23, and D44). Statistical comparisons between groups were performed and asterisks show significant value differences between indicated groups (red - increasing, black - decreasing): ${ }^{* *} p<0.001,{ }^{* *} p<0.01$ and ${ }^{*} p<0.05$.
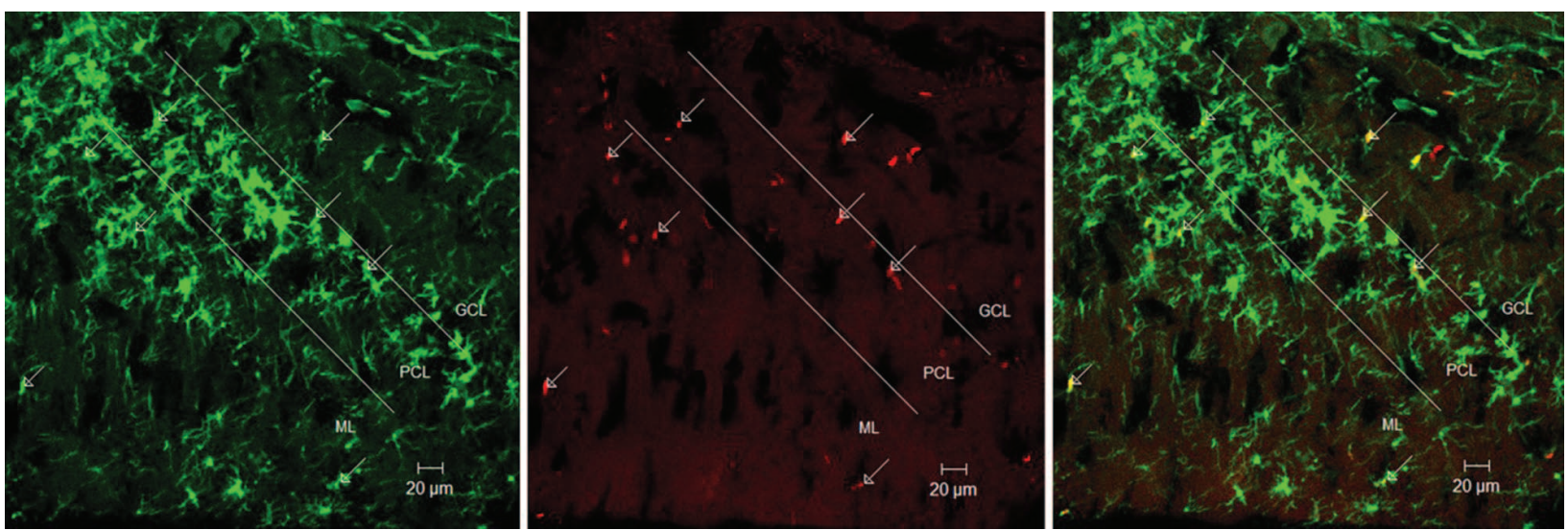

Fig. 2. Immunofluorescence double staining of BrdU+cells (red) and Iba1+ microglia (green) in the D4 group. Cells with colocalization of BrdU and Ibal markers (yellow, arrows) were found in all three layers of the primate cerebellar cortex. $M L$, molecular layer; PCL, Purkinje cell layer; GCL, granular cell layer. 

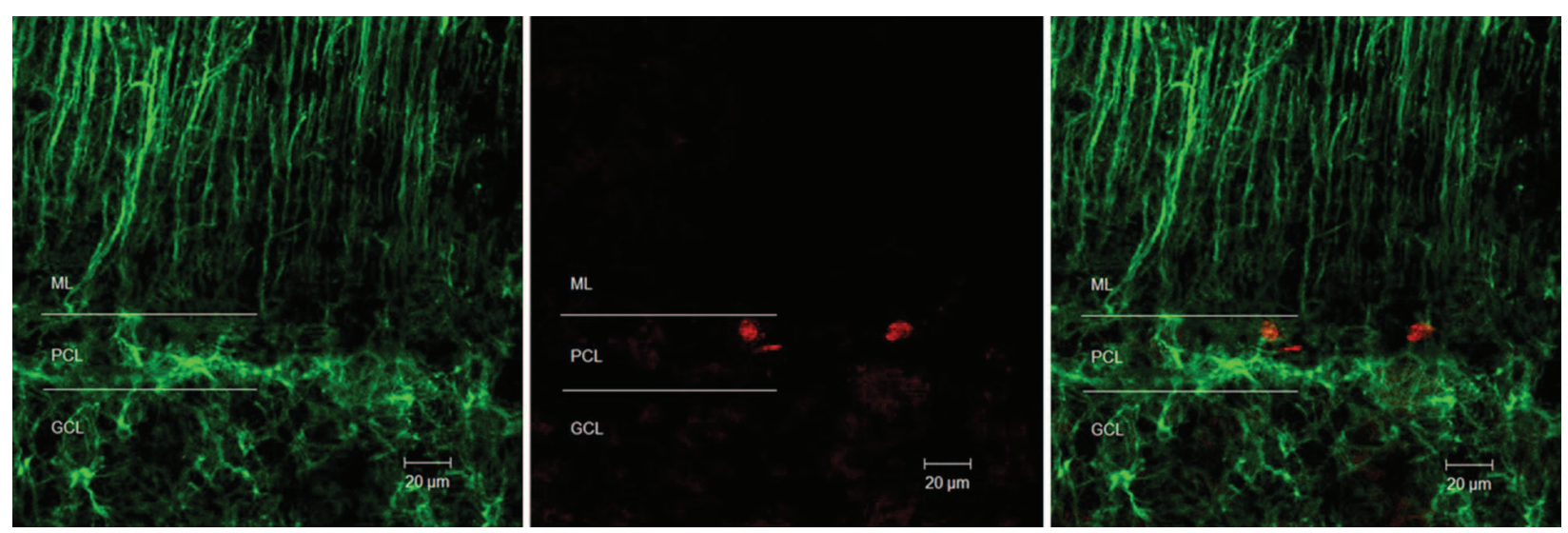

Fig. 3. Immunofluorescence double staining of BrdU+ cells (red) and GFAP+ astroglia (green) in the D9 group. No colocalization of BrdU and GFAP markers in any layers of the primate cerebellar cortex was found. ML, molecular layer; PCL, Purkinje cell layer, GCL, granular cell layer.

\section{RESULTS}

First, we counted all BrdU-labeled cells in determined layers/zones, i.e., molecular layer, granular layer, and white matter zone in the primate cerebrocerebellum (Fig. 1 a, b, c) and spinocerebellum (Fig. 1 $\mathrm{d}, \mathrm{e}, \mathrm{f}$ ) in all groups. Then, we statistically performed comparison between every two groups in each layer or zone. We found a significant increase in the molecular layer and white matter zone of the cerebrocerebellum in the D4, D9, and D44 groups, also a similar increase in all three regions of the spinocerebellum only in the D44 group. In most groups: D4, D9, D15, and D23, especially in spinocerebellum, a decrease was detected even if it was not statistically significant in each case.

Immunofluorescence double staining of BrdU+ cells and Iba1+ microglia revealed colocalization of these two markers in all layers investigated in most of the groups, including the D44 group, which was of the longest duration (Fig. 2).

There was no colocalization of the makers BrdU for dividing cells and GFAP for astroglia found in the cerebella of any group of this study. Bergmann glia were observed in large amounts at their typical location in the Purkinje cell layer. They exposed long immunopositive processes extending into the molecular layer up to the pial surface (Fig. 3).

\section{DISCUSSION}

In this study we investigated the effect of global brain ischemia on cell proliferation and differentiation in the primate cerebellum using BrdU and two other markers for specific cell types: Ibal for microglia and GFAP for astroglia. Ischemia was an important factor that stimulates cell division in the primate cerebellum. This finding corresponds to our previous data on other regions of the primate brain (18). The counts of BrdU-labeled cells showed increasing numbers in most of the groups studied, with the highest value in the D4 group for cerebrocerebellum, and in the D44 group - for spinocerebellum. This reflects the immediate effect of ischemia that was reduced in the next two groups, i.e. the D9 and D15 groups. This effect is most probably due to cell death of newly produced cells or decreasing amount of BrdU under the minimal threshold for detection following cell division. The D15 group showed the lowest number of BrdU+ cells in comparison with other time points. This group has a different BrdU paradigm starting at day 11 that may be responsible for the above-mentioned effect. The groups of longer ischemia duration, such as D23 and D44, demonstrated an increase in the numbers of BrdU+ cells in the cerebrocerebellum, in D44 - also in the spinocebellum. A plausible explanation may be the stable retention of BrdU taken up by a large number of newly generated cells during the initial days after an ischemic injury to the cerebellum. Such cells have survived, they contain the marker but have not divided yet or will not divide at all. Cells showing colocalization for BrdU and Ibal markers are newly generated microglia. We did not find any evidence for newly generated astroglia as colocalization for BrdU, and 
Vesselina Mihaleva, Stoyan Pavlov, Meglena Angelova et al.

GFAP was not detected. This result corresponds to similar observation of other researchers (16).

Additional labelings, using specific markers for neural stem/progenitor cells, are needed to specify the presence and phenotype of newly generated cells of the neuronal line.

\section{CONCLUSIONS}

Our results suggest that ischemia stimulates cellular proliferation in all zones of the primate cerebellum but this effect diminishes with the survival time after injury. Most of the newly produced cells differentiate into microglia or other non-neurogenic cell types. Our findings may expand the basic knowledge on tissue repair in the primate cerebellum after global brain ischemia and contribute to a better treatment of this condition in humans.

\section{REFERENCES:}

1. Basson MA, Wingate RJ. Congenital hypoplasia of the cerebellum: developmental causes and behavioral consequences. Front Neuroanat. 2013;7(29)1-15.

2. Butts T, Green MJ, Wingate RJ. Development of the cerebellum: simple steps to make a 'little brain'. Development. 2014;141(21):4031-4041.

3. Espinosa JS, Luo L. Timing neurogenesis and differentiation: insights from quantitative clonal analyses of cerebellar granule cells. J Neurosci. 2008;28(10):2301-2312.

4. Gage FH, Temple S. Neural stem cells: generating and regenerating the brain. Neuron. 2013;80(3):588-601.

5. Hashimoto M, Hibi M. Development and evolution of cerebellar neural circuits. Dev Growth Differ. 2012;54(3):373-389.

6. Juranek JK, Mukherjee K, Siddiqui TJ, Kaplan BJ, Li JY, Ahnert-Hilger G et al. Active zone protein expression changes at the key stages of cerebellar cortex neurogenesis in the rat. Acta Histochem. 2013;115(6):616-625.

7. Komuro H, Rakic P. Distinct modes of neuronal migration in different domains of developing cerebellar cortex. J Neurosci. 1998;18(4):1478-1490.

8. Lee A, Kessler JD, Read TA, Kaiser C, Corbeil D, Huttner WB et al. Isolation of neural stem cells from the postnatal cerebellum. Nat Neurosci. 2005 8(6):723-729.
9. Leto K, Bartolini A, Rossi F. The prospective white matter: an atypical neurogenic niche in the developing cerebellum. Arch Ital Biol. 2010;148(2):137-146.

10. Leto K, Rolando C, Rossi F. The genesis of cerebellar GABAergic neurons: fate potential and specification mechanisms. Front Neuroanat. 2012;6(6):1-10

11. Martinez S, Andreu A, Mecklenburg N, Echevarria D. Cellular and molecular basis of cerebellar development. Front Neuroanat. 2013;7:1-18.

12. Schilling K, Oberdick J, Rossi F, Baader SL. Besides Purkinje cells and granule neurons: an appraisal of the cell biology of the interneurons of the cerebellar cortex. Histochem Cell Biol. 2008;130(4):601-615.

13. Selvadurai HJ, Mason JO. Wnt $/ \beta$-catenin signalling is active in a highly dynamic pattern during development of the mouse cerebellum. PLoS One. 2011;6(8):e23012.

14. Seto $Y$, Ishiwata $S$, Hoshino M. Characterization of Olig2 expression during cerebellar development. Gene Expr Patterns. 2014;15(1):1-7.

15. Sotelo C. Cellular and genetic regulation of the development of the cerebellar system. Prog Neurobiol. 2004;72(5):295-339.

16. Su X, Guan W, Yu YC, Fu Y. Cerebellar stem cells do not produce neurons and astrocytes in adult mouse. Biochem Biophys Res Commun. 2014;450(1):378-383.

17. Tailor J, Kittappa R, Leto K, Gates M, Borel M, Paulsen O et al. Stem cells expanded from the human embryonic hindbrain stably retain regional specification and high neurogenic potency. J Neurosci. 2013;33(30):12407-12422.

18. Tonchev AB, Yamashima T, Zhao L, Okano HJ, Okano H. Proliferation of neural and neuronal progenitors after global brain ischemia in young adult macaque monkeys. Mol Cell Neurosci. 2003;23(2):292-301.

19. Yeung J, Ha TJ, Swanson DJ, Choi K, Tong Y, Goldowitz $\mathrm{D}$. Wls provides a new compartmental view of the rhombic lip in mouse cerebellar development. J Neurosci. 2014;34(37):12527-12537. 